\title{
Studying the social determinants of COVID-19 in a data vacuum
}

\author{
Kate H. Choi ${ }^{1}$ \\ Patrick Denice $^{1}$ \\ Michael Haan ${ }^{1}$ \\ Anna Zajacova ${ }^{1}$ \\ ${ }^{1}$ Department of Sociology, University of Western Ontario
}

Running title: COVID-19 data void in Canada

Keywords: COVID-19; neighborhood contexts; health inequality; racial inequality

Abstract: 174 words

Text excluding references, tables, and figures: 3,384 words

Number of references: 20

Figures: 3

Table: 1

Supplementary Information

The authors declare that they have no conflict of interest.

Funding: This work was supported by a SSHRC Insight Development Grant (430-2017-00920)

Corresponding author: Please direct all correspondence to Kate H. Choi, Department of Sociology, Social Science Centre 5331, University of Western Ontario, 1151 Richmond Street, London, Ontario, Canada, 519-661-2111 x-85115; hchoi228@uwo.ca

The final version of this paper was published in The Canadian Review of Sociology DOI:10.1111/cars.12336 


\title{
Studying the social determinants of COVID-19 in a data vacuum
}

\begin{abstract}
Objective: Many provinces in Canada are not collecting race and socio-demographic data on COVID-19 patients. Therefore, whether COVID-19 is disproportionately affecting certain sociodemographic groups is unknown. We make creative use of existing data to fill this data vacuum and identify the socio-demographic risk factors for COVID-19 in Canadian health regions. We use insights from these analyses to identify potential COVID-19 for smaller geographic units. Methods: We merge COVID-19 data with tabular census data. We use Poisson regression models to predict cumulative counts of COVID-19 infection in health regions in Canada. We identify potential COVID-19 hotspots by combining the insights from the analysis of risk factors of COVID-19 in health regions with information on the socio-demographic profiles for smaller geographic units.
\end{abstract}

Results: COVID-19 infection and mortality rates are higher in communities with larger shares of Black, refugee, and low-income residents.

Conclusion: Our approach offers a way for researchers and policymakers to make creative use of existing data to identify communities vulnerable to pandemics in the absence of granular data about the spread of infection. 


\section{Significance Statement}

The Canadian government is not making race and socioeconomic data on individual COVID-19 patients to researchers. There hasn't been a systematic study that has examine whether racial minorities, immigrants, and low-income individuals are particularly vulnerable to COVID-19. We make creative use of existing data and identify the socio-demographic risk factors for COVID-19 in health regions. In Canada, the smallest geographic unit for which COVID-19 data is consistently available the health region, which is too large and too heterogeneous to capture the spread of COVID-19 in local communities. Using the results from our analyses of health regions and information about the socio-demographic traits of census subdivisions, we identify hotspots for smaller geographic units. Our approach predicted several locations which later became hotspots. Such an approach may offer a way for researchers and governments to identify hotspots in the absence of infectious data at the local level. 


\section{Introduction}

Racial/ethnic minorities and low-income groups in the U.S. and the U.K. are particularly vulnerable to COVID-19 (Chin et al. 2020; Thebault et al. 2020). Whether this is also true in Canada is unknown because researchers do not have access to data about the race/ethnicity or socioeconomic status of COVID-19 patients (Ramos 2020; Siddiqi et al. 2020). Despite repeated pleas for such data from social scientists, government officials initially responded that they have no plans to make these data available because all groups are important and it may violate the privacy of patients (Mulligan et al., 2020; Osman, 2020; Perkel, 2020). With mounting social pressure, a subset of provincial governments are only now considering the possibility of making this data available to researchers (Perkel, 2020).

Canadian researchers are primarily relying on two data sources to examine the pattern of spread of COVID-19. The first comes from the Public Health Agency of Canada (PHAC). These data report the daily infection and death counts for 87 health regions, but they are not tallied by race or socioeconomic status. Furthermore, each health region encompasses approximately 415,000 residents; thus, they are too large geographically and too heterogeneous socio-demographically to capture the impact of COVID-19 on local communities and specific sub-populations. The second is crowdsourced data, which relies on self-reports of COVID-19 cases and collects individuals' postal code (Jones 2020). While postal code areas are small enough to represent communities, these data likely miss a significant portion of COVID-19 cases by relying on individuals' access to and willingness to report their symptoms on internet sites. These data have been used to identify certain vulnerable communities — specifically, those who live in densely populated areas or places 
with higher shares of older (70+) residents - but they ignore the community's racial and socioeconomic composition.

Whether or not Black, immigrant, and low-income communities in Canada have been disproportionately affected by COVID-19 is a priori unclear. Like in the U.S., Blacks and immigrants in Canada are more economically disadvantaged than whites (Attewell et al. 2010). The economically disadvantaged are more likely to work in frontline occupations facing greater exposure to COVID-19. There are, however, reasons to believe that the pattern of the COVID-19 spread will differ across the two countries. Unlike the U.S., Canada has universal health insurance, and Black Canadians generally report health statuses comparable to whites, partially reflecting the fact that large shares of Black Canadians are high-skilled immigrants and positively selected in terms of health (Lebrun \& LaVeist 2013).

We fill this data void by linking aggregated COVID-19 data with tabular census data detailing the socio-demographic profile of health regions and communities. We present exploratory analyses identifying the risk factors for COVID-19 infection and mortality rates in health regions. Results from this analysis are combined with information about the sociodemographic traits of smaller communities to identify potential COVID-19 hotspots on a more granular geographic level. It is worth noting that our results are descriptive in nature and our analysis is not meant to establish "causality".

\section{Methods}

\section{Data and Sample}

We obtained daily counts of COVID-19 infections and deaths through May 15 for each health region from the University of Toronto's COVID-19 Canada Open Data Working Group 
(Berry et al. 2020). ${ }^{1}$ We focus on 84 (out of 87) health regions. We exclude 3 regions in Yukon, the Northwest Territories, and Nunavut because they are sparsely populated and report virtually no cases.

We combined the COVID-19 counts with tabular data estimated from the 2016 Canadian Census. These data, downloaded from the Statistics Canada website, report the socio-demographic profiles of health regions, census subdivisions (CSDs), and census divisions (CDs). ${ }^{2}$ CDs are similar to U.S. counties. CSDs, which are nested within CDs, are similar to U.S. towns or small cities. Due to privacy concerns, Statistics Canada suppresses information when the tallied counts of CSD residents belonging to a socio-demographic category are small. For example, if the number of Black residents in a CSD is less than 10, the value is suppressed. To minimize biases that come from suppressed data, we defined communities as CSDs with more than 2,000 residents or remaining areas within the CD outside of these populous CSDs. With a median population of 6,929 , these geographic areas are smaller and represent more localized communities than health regions. There are 1,202 communities: 943 CSDs and 259 CD-specific areas outside of these CSDs.

\section{Measures}

Our outcomes are cumulative counts of COVID-19 infections and deaths through May 15, 2020, in each health region. We focused on this date because Canadian provinces started to re-open their economies around this time.

\footnotetext{
${ }^{1}$ In the supplementary information, we include estimates using daily counts until June 15, 2020 and obtain similar results. ${ }^{2}$ https://www12.statcan.gc.ca/census-recensement/2016/dp-pd/prof/details/downloadtelecharger/comp/page_dl-tc.cfm?Lang=E
} 
Our covariates are percent Black, percent foreign-born, percent refugees, percent without proficiency in English/French, percent poor, percent with a college degree, percent of residents 65 years and older, percent of workers in health-care, percent of workers in agriculture, ${ }^{3}$ and the size of the geographic area. ${ }^{4}$ Studies in other countries have identified them as potential risk (or protective) factors for COVID-19 (Thebault 2020; Yancy 2020). Poor residents are defined as those whose income falls below Statistics Canada's lowincome cut-off (LICO), which is akin to the U.S. poverty line. These measures are computed for health regions and communities by Statistics Canada. We exclude other measures capturing socio-demographic composition (e.g., percent Asian) due to multicollinearity (VIF>10). Earlier models also included percent Indigenous. We exclude this covariate from our analyses for two reasons. According to media reports, COVID-19 infections and deaths of Indigenous people have been vastly undercounted (Patel 2020). Moreover, preliminary analyses revealed that our conclusions were sensitive to the definition used for Indigenous people (i.e., official registration, sole ancestry, primary language used at home).

\section{Analytical strategy}

We first identify the risk factors for COVID-19 infection and mortality rates in health regions using Poisson regression with the exposure command. Poisson regression estimates COVID-19 counts, and the exposure option divides the estimated counts by the population size. We present incidence rate ratios $\left(\mathrm{e}^{\beta}\right)$ to describe the association between the socio-demographic traits of health regions and COVID-19 infection and mortality rates.

\footnotetext{
${ }^{3}$ This measure captures rural/urban status.

${ }^{4}$ After we divide by population size, it is the inverse of population density and captures low density.
} 
We present the results using standardized covariates in the main text and unstandardized covariates in the Supplementary Information.

We then identify potential COVID-19 hotspots by predicting the relative number and rate of COVID-19 infections and deaths at a more granular geographic level than the health regions. We predict these rates and counts using (a) the coefficients describing the association between the social, demographic, and economic composition of health regions presented in Table S3, and (b) information about the socio-demographic composition of these smaller communities. We then use the predicted counts as weights to partition the actual number of COVID-19 infections/deaths in a health region across its constituent communities. ${ }^{5}$ For these analyses, we use unstandardized covariates because the distribution of these covariates differs across health regions and communities. In our discussion of potential COVID-19 hotspots, we discuss mainly outbreaks identified by media reports.

\section{Results}

\section{Descriptive results}

Figure 1 presents the spatial distribution of Black, immigrant, and low-income communities in Canada. Communities with higher shares of Black and immigrant residents are located in Canada's three largest cities: Montreal, Toronto, and Vancouver. Outside of these cities, Black communities are in northeast Alberta and northern Quebec. Immigrant communities

\footnotetext{
${ }^{5}$ Toronto, Montreal, and Alberta released official COVID-19 counts for geographic units smaller than health regions. Starting in mid-March, Alberta released daily active (but not new or cumulative) counts of COVID-19 infections for public health authorities, which are subdivisions of health regions. Starting on May 25, 2020, Toronto reported the cumulative infection (but not death) counts. Daily "new" (but not cumulative) counts have been available in Montreal boroughs from mid-March. Inconsistencies in data collection and reporting preclude us from observing COVID-19 data for regions smaller than public health regions.
} 
are also located throughout British Columbia, in southern Alberta, in northeast Alberta, and in southern Ontario. Low-income communities are found in northern Saskatchewan, northern Manitoba, and southern Ontario.

Figure 1 goes here.

Figure 2 shows the spatial distribution of COVID-19 infection counts and rates across the 84 health regions in our analysis. COVID-19 count rates and counts are high in Montreal, Toronto, Vancouver, southern Alberta including Calgary, the corridor between Montreal and Quebec City, and southwestern Ontario bordering Detroit. In some places, high rates and counts are not coincident. Saskatchewan and northern Alberta have high COVID-19 infection and death counts, but not high rates. Northern Quebec has high COVID-19 infection/mortality rates, but not high counts.

Figure 2 goes here.

\section{Multivariate analysis}

Panel A in Table 1 presents the results from Poisson regression models predicting the COVID-19 infection rates for the 84 health regions. COVID-19 infection rates are higher in health regions with higher shares of Black residents, refugees, immigrants without English/French proficiency, lower-income individuals, adults aged 65+ years, and health-care workers. For example, net of other characteristics, COVID-19 infection rates double with a 1 standard deviation increase in the share of Black residents in a health region. By contrast, COVID-19 infection rates are lower in low-density regions with higher shares of non-refugee immigrants and workers in the agricultural industry. For example, net of other characteristics, a 1 standard deviation increase in the share of agricultural workers is associated with a $15 \%$ drop in COVID-19 infection rates. 


\section{Table 1 goes here.}

Panel B presents results from analogous analyses predicting COVID-19 mortality rates. The results for COVID-19 mortality rates are similar to those presented in Panel A. Two exceptions deserve mention. The share of adults 65 years and older in health regions has much stronger implications for COVID-19 mortality rates than for COVID-19 infection rates. A 1 standard deviation increase in the share of adults 65 years and older in health regions is associated with a 9\% increase in COVID-19 infection rates. By contrast, the same change increased COVID-19 mortality rates by a factor of 2.1 times. Another exception is that COVID19 mortality rates are lower in health regions with higher shares of workers in the healthcare industry. A 1 standard deviation increase in the share of health care workers in the health region is associated with a 19\% decrease in COVID-19 mortality rates. Differences in COVID-19 infection rates by the share of healthcare workers in the health region are minimal. Greater availability of healthcare personnel in one's place of residence may mean that patients receive the appropriate treatment when infected with the virus.

\section{Potential COVID-19 hotspots at a more granular geographic level}

Figure 3 displays the predicted COVID-19 infection and death counts and rates across the 1,202 communities (i.e., CSDs and CDs). ${ }^{6}$ A comparison of Figures 2 and 3 reveals that considerable heterogeneity exists in the spread of the COVID-19 within health regions. As mentioned earlier, we focus on the potential COVID-19 hotspots identified in media reports as the location of COVID-19 outbreaks. By focusing on a lower geographic level, we find urban/rural differences in the spread of COVID-19 between southwest Alberta near Calgary and southeast Alberta. Northeastern (but not the rest of) Alberta and northern (but not southern)

\footnotetext{
${ }^{6}$ Local newspapers have reported COVID-19 outbreaks in most of these communities.
} 
Saskatchewan have seen higher numbers of infections and deaths from COVID-19. These two regions are the home to large numbers of temporary migrant workers living in tight quarters while they work in the oil sands. Figure 3 also highlights high COVID-19 counts and rates in Southern Ontario along the border with Michigan and Minnesota, which are communities with high shares of Black, immigrant, and low-income populations.

Figure 3 goes here.

We also found that our understanding of the geographic spread of COVID-19 differs depending on how we measure its severity. Focusing on counts reveals COVID-19 hotspots in northeastern Alberta and northern Saskatchewan. Focusing on rates, however, highlights hotspots along the Canadian-U.S. border in the southeastern corridor between Toronto and Montreal, in British Columbia, and in eastern Manitoba. These hotspots may emerge due to U.S.Canadian differences in the spread and containment of the virus (Coletta, 2020).

\section{Discussion}

The absence of socio-demographic data about COVID-19 patients prevents researchers from determining whether the pandemic is disproportionately affecting certain sociodemographic groups in Canada. We fill this void by pooling COVID-19 data with tabular census data to (a) identify the risk factors associated with COVID-19's spread and (b) pinpoint potential COVID-19 hotspots in smaller geographic communities.

Like in the U.S. and U.K, Black communities in Canada have been disproportionately impacted by COVID-19. This may explain why places like Montreal, with large numbers of Black immigrants, have emerged as Canada's COVID-19 epicenters. In a way, these results are surprising given that discrimination against Black communities is thought to be less severe in Canada and Black immigrants are typically younger than Canadian-born. It also underscores the 
importance of examining racial and ethnic inequalities in health in the Canadian context where many public health officials and politicians have not seen the need to collect racial data on grounds that documenting health inequality by race/ethnicity may lead to discrimination (Siddiqi et al., 2020; Mulligan et al., 2020).

Immigrant groups' vulnerability to COVID-19 may vary depending on their admission status and proficiency in the destination country's official languages. The zero-order association between the percentage foreign-born in a health region and COVID-19 infection/mortality rates is positive (in Table 2). This association, however, becomes negative when we control for the share of refugees and of immigrants without English/French proficiency. The adjusted association between share of refugees and COVID-19 infection/mortality rates is positive, as is its association with the share of immigrants without English/French proficiency. Prior to their arrival in Canada, refugees may have spent years in refugee camps with limited access to healthcare. Immigrants with limited proficiency in the destination country's language have lower healthcare usage (Kirkman and Mondragon 1991). Both groups of immigrants may have more pre-existing health conditions that render them more vulnerable to COVID-19. Other immigrants to Canada may be less vulnerable to COVID-19 given their generally higher skill and age.

A higher share of adults 65 years and older in a health region is associated with higher COVID-19 infections and especially deaths. These results are consistent with research showing that COVID-19 infections are more lethal among the elderly (Liu et al. 2020) and news reports showing that a significant portion of COVID-19-related deaths in Canada occurred in long-term care facilities for the elderly (MacCharles 2020).

We also show that collecting COVID-19 data at larger units of geography (e.g., health regions) masks important heterogeneity within these areas, such as urban/rural differences in the 
severity of the spread in COVID-19. It may also hide the impact of COVID-19 in industries that employ large numbers of immigrant workers and that house them in group quarters, including those in northeastern Alberta and north Saskatchewan which saw alarming COVID-19 outbreaks in early May (Taylor, 2020). It is worth noting that our statistical models were able to identify several communities which experienced outbreaks of COVID-19 infections. Our methodological approach may offer researchers and public health officials a way to identify communities vulnerable to COVID-19 ahead of time and take preventative measures to ensure that the pandemic does not spread to these vulnerable communities even in the absence of granular data. Finally, we show that our understanding of the severity of the spread of COVID-19 infections/deaths may differ depending on how we measure it (i.e., rates and counts). Currently, the Canadian government and media focus on counts. Examining rates reveals several COVID hotspots near border towns with the U.S., some of which are sparsely populated but have high shares of residents infected with COVID-19. In focusing on counts, policymakers may be missing these potential COVID-19 hotspots.

Our study has a few limitations. We rely on aggregate data about the socio-demographic traits of communities to infer traits about individual residents. We recognize that this approach could lead to ecological fallacies. However, only a subset of Canadian provinces has started to collect data on individual COVID-19 patients and has plans to make this data available to researchers. Even if these provinces release data as planned, Canadian researchers will have access to official data on individual COVID-19 patients in late 2020 (Allen 2020). Many public health researchers expect a second wave of COVID-19 in the fall of 2020. Insights about the social determinants of COVID-19 are necessary to ensure that local governments take the preventative steps necessary to reduce the impact of the second wave of COVID-19. 
Furthermore, even if individual data become available, privacy laws in Canada may delay and even prevent researchers from getting access to race data on individual COVID-19 patients. Our approach indirectly fulfills both needs. Second, "health region" is the smallest geographic unit for which COVID-19 data are consistently available across Canada. When we computed the predicted infection/mortality rates, we assumed that the risk factors associated with COVID-19 infections/deaths do not vary across communities within health regions. It is, however, worth noting that our approach identified several outbreak sites before it was the subject of media reports; and as such, this approach may offer researchers and policymakers a way to identify potential hotspots when confronted with data gaps about the spread of a pandemic at the local level. Third, our reliance on public-use tabular census data precludes us from considering several potentially vulnerable groups, such as temporary migrants and the age- and gender-specific distributions of different populations. Finally, we rely on official counts of COVID-19 cases. Borjas (2020) found that testing rates were lower among residents of minority and low-income neighbourhoods in New York City. We may underestimate the severity of COVID-19 in such communities in Canada. 


\section{References}

Allen, K. (2020, July 2). Ontario is starting to collect race-based COVID-19 data. Some worry that it could do more harm than good. Toronto Star. https://www.thestar.com/news/gta/2020/07/02/ontario-is-starting-to-collect-race-basedcovid-19-data-some-worry-it-could-do-more-harm-than-good.html

Attewell, P., Kasinitz, P., \& Dunn, K. (2010). Black Canadians and black Americans: racial income inequality in comparative perspective. Ethnic and Racial Studies 33(3): 473-495. DOI: $10.1080 / 01419870903085883$

Borjas, G. (2020). Demographic Determinants of Testing Incidence and COVID-19 Infections in New York City Neighborhoods. NBER Working Paper No. 26952.

Berry, I., Soucy, J., Tuite, A. \& Fisman, D. (2020). Open access epidemiologic data and an interactive dashboard to monitor the COVID-19 outbreak in Canada. Canadian Medical Association Journal 192 (15): E420. https://doi.org/10.1503/cmaj.75262

Chin, T., R. Kahn, R. Li, J. Chen, N. Krieger, C. Buckee, S. Balsari, \& Kiang, M. (2020). U.S. county-level characteristics to inform equitable COVID-19 response. MedRxiv https://doi.org/10.1101/2020.04.08.20058248.

Coletta, A. (2020, July 3). Some Canadian businesses want to let Americans back in. Most Canadians don't. The Washington Post. https://www.washingtonpost.com/world/the_americas/us-canada-bordercoronavirus/2020/06/30/2cbfd3b0-b55c-11ea-a510-55bf26485c93_story.html

Jones, A. (2020, March 27). Canadian university students create map of self-reported potential COVID-19 cases. $\boldsymbol{C T V}$ News. https://www.ctvnews.ca/sci-tech/canadian-university$\underline{\text { students-create-map-of-self-reported-potential-covid-19-cases-1.4872245 }}$ 
Kirkman-Liff, B. and D. Mondragon. (1991). Language of interview: relevance for research of Southwest Hispanics. American Journal of Public Health 81: 1399-1404.

Lebrun, L. A., \& LaVeist, T. A. (2013). "Health status among Black Canadians: Results from a national survey." Canadian Ethnic Studies, 45(1-2): 143-155.

Liu, K., Y. Chen, R. Lin, \& Han, K. (2020). “Clinical features of COVID-19 in elderly patients: A comparison with young and middle-aged patients". The Journal of Infection. 10.1016/j.jinf.2020.03.005

MacCharles, Tonda. (2020, May 7). 82\% Canada's COVID-19 deaths have been in long-term care, new data reveals. Toronto Star. https://www.thestar.com/politics/federal/2020/05/07/82-of-canadas-covid-19-deaths-havebeen-in-long-term-care.html

Mulligan, K., J. Rayner, and Oyne Nnorom. (2020, April 30). Race based data urgently needed during the pandemic. The Conversation. https://theconversation.com/race-based-healthdata-urgently-needed-during-the-coronavirus-pandemic-136822.

Osman, L. (2020, May 6). Canada still only considering gathering race-based COVID-19 data. CTV News. https://www.ctvnews.ca/health/coronavirus/canada-still-only-consideringgathering-race-based-covid-19-data-1.4927648

Patel, R. (2020, May 7). Canada must improve COVID-19 data collection for Indigenous communities, minister says. $\boldsymbol{C B C}$ News. https://www.cbc.ca/news/politics/indigenous$\underline{\text { covid-19-data-collection-1.5563433 }}$

Perkel, C. (2020, June 2). Ontario mulls collection of race-based coronavirus data; some argue it's essential. Global TV News. https://globalnews.ca/news/7018459/ontario-coronavirus$\underline{\text { race-based-data/ }}$ 
Ramos, H. (2020, April 14). Being Counted in Canada's Coronavirus Data: Stronger data collection is essential to understand how COVID-19 impacts immigrants across Canada. New Canadian Media: The Pulse of Immigrant Canada.

https://newcanadianmedia.ca/being-counted-in-canadas-coronavirus-data/

Siddiqi, A., A. Blair, and A. Parnia. (2020, April 16). A lack of data hides the unequal burden of COVID-19. Toronto Star. https://www.thestar.com/opinion/contributors/2020/04/16/a-lackof-data-hides-the-unequal-burden-of-covid-19.html

Taylor, S. (2020, May 6). COVID 19 outbreak in northern Saskatchewan 'of concern' says Canada's chief health officer. Global TV News. https://globalnews.ca/news/6912579/covid-19-outbreak-saskatchewan-dr-theresa-tam/ Thebault, R., Ba Tran, A., \& Williams, V. (2020, April 7). The coronavirus is infecting and killing black Americans at an alarmingly high rate. Washington Post. https://www.washingtonpost.com/nation/2020/04/07/coronavirus-is-infecting-killing-blackamericans-an-alarmingly-high-rate-post-analysis-shows/?arc404=true.

Yancy, C. (2020). COVID-19 and African Americans. JAMA. Doi: 10.1001/jama.2020.6548 


\section{Figures}

Figure 1. Community Characteristics
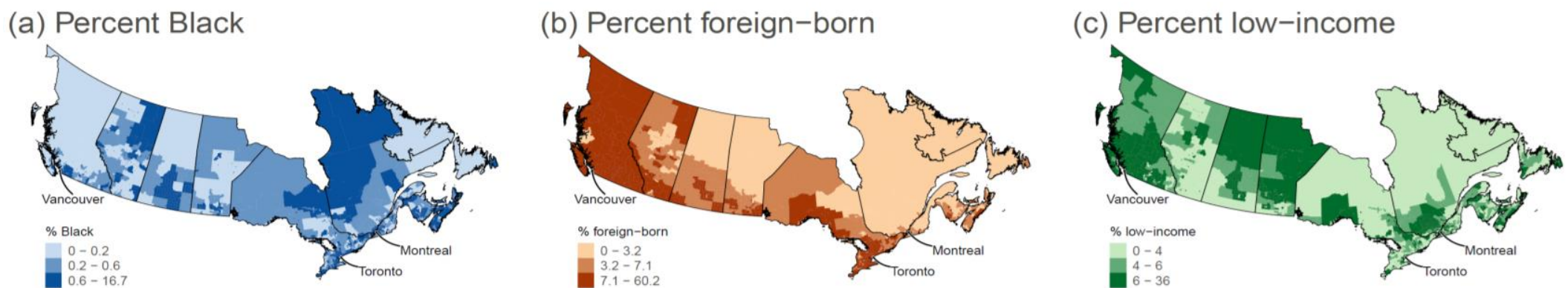

Source: Tabular data from Statistics Canada computed from the 2016 Census Sample: 1,202 CSDs/CDs 
Figure 2. COVID-19 infection and death counts in health regions
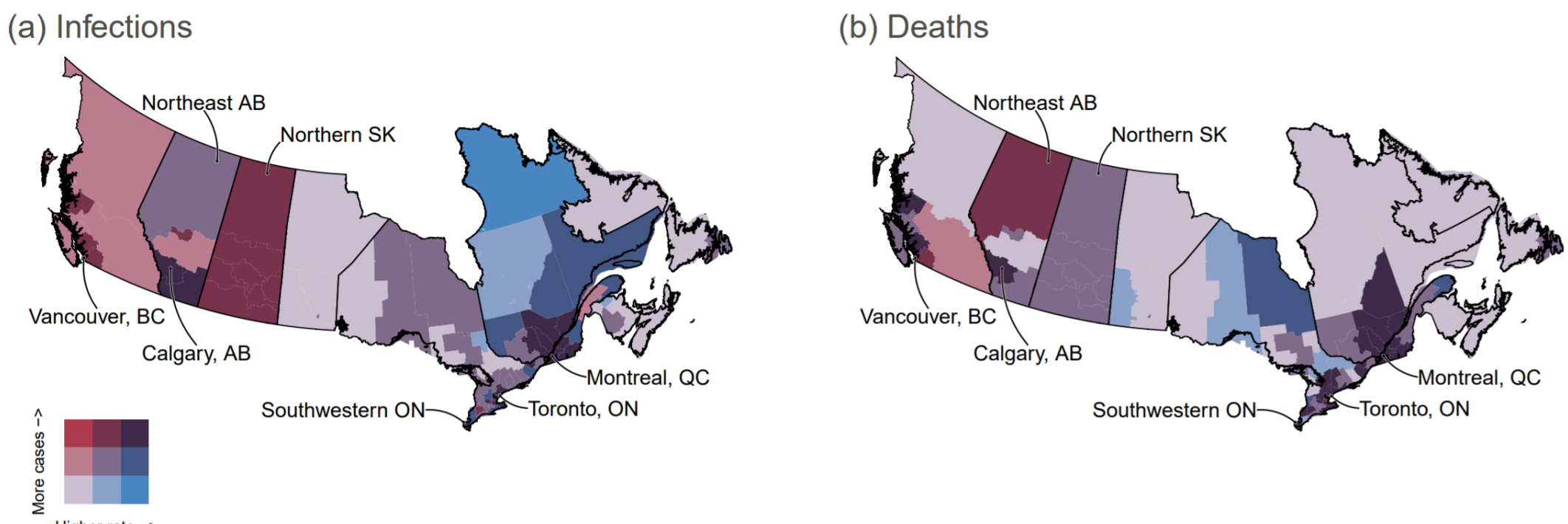

Sources: University of Toronto's COVID-19 Canada Open Data Working Group Dashboard and tabular data from Statistics Canada estimated from 2016 Canadian Census

Sample: 84 health regions in Canada

Notes:

- Rates are COVID-19 infection/death counts/100,000 residents

- We divide health regions into 9 cross-classified categories based on the terciles of counts and rates.

For infection counts, the terciles are bottom (1-39), middle (39-471), and top (471-22,028).

- For infection rates, the terciles are bottom (1-26), middle (26-107), and top (107-1,134).

- For death counts, the terciles are bottom (0-1), middle (1-13), and top (13-2,296).

- For mortality rates, the terciles are bottom (0-1), middle (1-5), and top (5-118). 
Figure 3. Predicted COVID-19 infection and mortality rates in communities
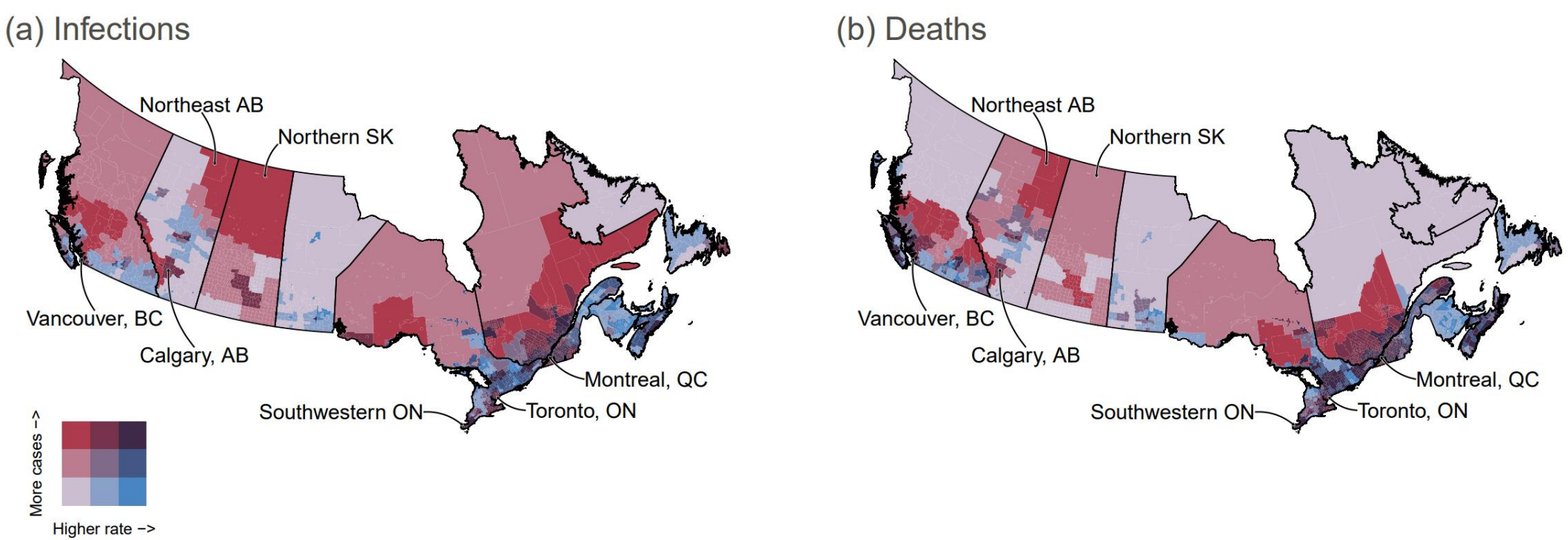

Sources: University of Toronto's COVID-19 Canada Open Data Working Group Dashboard and tabular data from Statistics Canada estimated from 2016 Canadian Census

Sample: 1,202 CSDs/CDs in Canada

Notes:

- Rates are COVID-19 infection/death counts/100,000 residents

- We divide health regions into 9 cross-classified categories based on the terciles of counts and rates.

- For infection counts, the terciles are bottom (1-11), middle (11-74), and top (74-4,985).

- For infection rates, the terciles are bottom (0-3), middle (3-15), and top (15-22,028).

- For death counts, the terciles are bottom (0-.01), middle (0.01-1.8), and top (1.8-5,920).

- For mortality rates, the terciles are bottom (0-0.007), middle (0.007-.180), and top (.181-2,296) 


\section{Tables}

Table 1. Poisson Regression Models Estimating Infection and Mortality Rates

\begin{tabular}{|c|c|c|c|c|c|c|}
\hline \multirow{2}{*}{$\begin{array}{r}\text { Characteristics of health regions/ } \\
\text { /Standardized covariates }\end{array}$} & \multicolumn{3}{|c|}{ Infection rates } & \multicolumn{3}{|c|}{ Mortality rates } \\
\hline & IRR & & $\beta / \mathrm{se}$ & IRR & & $\beta / \mathrm{se}$ \\
\hline$\%$ Black & 2.04 & $* * *$ & 138.16 & 2.56 & $* * *$ & 39.08 \\
\hline$\%$ Foreign-born & 0.53 & $* * *$ & -50.26 & 0.51 & $* * *$ & -11.03 \\
\hline$\%$ Refugees & 1.05 & $* * *$ & 9.40 & 1.13 & $* * *$ & 5.31 \\
\hline$\%$ Neither proficient in English/French & 1.35 & $* * *$ & 26.70 & 1.22 & $* *$ & 3.03 \\
\hline$\%$ Poor & 1.07 & $* * *$ & 11.45 & 1.23 & $* * *$ & 8.02 \\
\hline$\%$ Residents w/ college degree & 0.93 & $* * *$ & -10.96 & 0.84 & $* * *$ & -6.17 \\
\hline$\%$ Residents 65 years and older & 1.09 & $* * *$ & 10.00 & 2.08 & $* * *$ & 17.58 \\
\hline$\%$ Health-care workers & 1.01 & & 0.99 & 0.81 & $* * *$ & -4.04 \\
\hline$\%$ Agricultural workers & 0.78 & $* * *$ & -22.48 & 0.51 & $* * *$ & -11.35 \\
\hline Lower density & 0.85 & $* * *$ & -17.19 & 0.64 & $* * *$ & -5.42 \\
\hline Intercept & 0.00 & $* * *$ & -940.41 & 0.00 & $* * *$ & -211.34 \\
\hline$N$ & \multicolumn{6}{|c|}{84} \\
\hline Pseudo- $R^{2}$ & \multicolumn{3}{|c|}{0.60} & \multicolumn{3}{|c|}{0.73} \\
\hline
\end{tabular}

Sources: University of Toronto's COVID-19 Canada Open Data Working Group Dashboard and tabular data from Statistics Canada estimated from 2016 Canadian Census Sample: 84 health regions in Canada Notes: Offsets for population size. IRR denotes incidence ratio rates $\left(\mathrm{e}^{\beta}\right) . * * * \mathrm{p}<0.001$; $* * \mathrm{p}<0.01 ;{ }^{*} \mathrm{p}<0.05$ 


\section{Supporting information for}

"Studying the social determinants of COVID-19 in a data vacuum"

Kate H. Choi ${ }^{1}$

Patrick Denice ${ }^{1}$

Michael Haan ${ }^{1}$

Anna Zajacova ${ }^{1}$

${ }^{1}$ Department of Sociology, University of Western Ontario

Corresponding author: Kate H. Choi

E-mail: $\underline{\text { hchoi228@uwo.ca }}$

\section{This file includes}

Supplementary text

Tables S1 to S5 


\section{SUPPLEMENTARY TEXT}

We ran several models to test the robustness of our results. Results from supplementary analyses are presented in the Appendix. First, in Canada, provinces have the legal mandate to collect health data and administer healthcare for its residents. ${ }^{7}$ COVID-19 infection and death counts vary significantly across provinces, which may be partially the product of provincial differences in COVID-19 responses. We wanted to include a series of province fixed effects, but these models estimating COVID-19 mortality rates fail to converge because disproportionately large numbers of COVID-19 deaths occurred in Quebec. The inclusion of these province fixed effects alters our results for COVID-19 infections in two meaningful ways. First, the coefficients capturing the association between the social, economic, and demographic profile of health regions and COVID-19 infection rates are smaller once we add these covariates. Second, the association between the percent foreign-born and COVID-19 infection rates is negative in the absence of controls for provinces but is positive after we control for province. This is largely because economic class immigrants are disproportionately present in the province of British Columbia, which had lower COVID-19 infection rates. These results are available upon request.

Second, in the main text, we present results using standardized covariates to examine the association between the social, demographic, and economic composition of health regions and COVID-19 infection/mortality rates. These sample characteristics are presented in Table S2. We also ran the analyses using the actual percentages. These results, provided in Table S3, are substantively consistent with those presented in the main article.

\footnotetext{
${ }^{7}$ https://www.canada.ca/en/health-canada/services/canada-health-care-system.html
} 
Third, we tested the robustness of our conclusions by running the same models for several stages of the pandemic: April 15 (i.e., roughly the peak) and June 15 (i.e., close to the second phase of re-opening in several COVID-19 epicenters). Table S4 presents these results. Our substantive conclusions are robust, regardless of the timeframe examined.

Fourth, Ontario and Quebec have been the two provinces in Canada with the most COVID-19 cases. These provinces also have large black and immigrant populations. A potential concern may be that the association between socio-demographic profiles and COVID-19 infection/mortality rates in these two provinces are driving our results: COVID19 infection rates are higher in black and immigrant communities in these provinces, but such an association does not exist elsewhere. To test this premise, ideally, we would have liked to run our analyses for health regions outside of Ontario and Quebec. There are, however, only 32 health regions outside of these provinces, rendering the sample too small for us to obtain reliable estimates. Therefore, we ran robustness checks for the 52 health regions in Ontario and Quebec. The coefficients for the association between the share of black residents and COVID-19 infection and mortality rates are smaller for these supplementary analyses than they are for the analyses presented in the main text. The same is also true for supplementary analyses examining the association between the share of immigrants and COVID-19 infection and mortality rates. These findings suggest that (a) the national results are not the 'watered down' version of the Ontario and Quebec results and (b) such an association likely exists in Canadian provinces outside of Ontario and Quebec. Table S5 presents the results for health regions in Ontario/Quebec and all other provinces. Finally, we ran robustness checks with COVID-19 data for the 140 neighborhoods in Toronto. Our main results are robust. The only exception is that percent in the 
agricultural industry is positively correlated with COVID-19 infection rates. This differential likely emerges because percent in the agricultural industry ceases to represent urban/rural status for residents of the City of Toronto. These results are available upon request. The overall implication is that the associational patterns also hold when we examine how socio-demographic composition affects COVID-19 infection/mortality rates at a more granular level. 
Table S1. Poisson Regression Predicting Infection and Mortality Rates (Zero-order associations)

\begin{tabular}{|c|c|c|c|c|c|c|}
\hline \multirow{2}{*}{$\begin{array}{r}\text { Characteristics of health regions/ } \\
\text { /Standardized covariates }\end{array}$} & \multicolumn{3}{|c|}{ Infection rates } & \multicolumn{3}{|c|}{ Mortality rates } \\
\hline & IRR & & $\beta / \mathrm{se}$ & IRR & & $\beta / \mathrm{se}$ \\
\hline$\%$ Black & 1.56 & $* * *$ & 201.70 & 1.85 & $* * *$ & 71.24 \\
\hline$\%$ Foreign-born & 1.27 & $* * *$ & 92.32 & 1.42 & $* * *$ & 37.07 \\
\hline$\%$ Refugees & 1.28 & $* * *$ & 68.86 & 1.32 & $* * *$ & 21.01 \\
\hline$\%$ Neither proficient in English/French & 1.17 & $* * *$ & 45.48 & 1.27 & $* * *$ & 19.36 \\
\hline$\%$ Poor & 1.02 & $* * *$ & 8.21 & 1.10 & $* * *$ & 11.96 \\
\hline$\%$ Residents w/ college degree & 1.41 & $* * *$ & 111.17 & 1.67 & $* *$ & 43.70 \\
\hline$\%$ Residents 65 years and older & 0.83 & $* * *$ & -39.12 & 0.91 & $* * *$ & -5.83 \\
\hline$\%$ Health-care workers & 0.87 & $* * *$ & -24.21 & 0.84 & $* * *$ & -8.29 \\
\hline$\%$ Agricultural worker & 0.41 & $* * *$ & -118.50 & 0.19 & $* * *$ & -40.97 \\
\hline Lower density (land size) & 0.45 & $* * *$ & -64.94 & 0.02 & $* * *$ & -29.08 \\
\hline$N$ & \multicolumn{6}{|c|}{84} \\
\hline
\end{tabular}

Source: University of Toronto's COVID-19 Canada Open Data Working Group Dashboard and tabular data from Statistics Canada estimated from 2016 Canadian Census

Sample: 84 health regions in Canada

Notes: Offsets for population size. ${ }^{* * *} \mathrm{p}<0.001 ; * * \mathrm{p}<0.01 ; * \mathrm{p}<0.05$ 
Table S2. Descriptive Analysis

\begin{tabular}{|c|c|c|}
\hline \multirow{2}{*}{\multicolumn{3}{|c|}{ A. Characteristics of health regions }} \\
\hline & & \\
\hline$\%$ Black & 1.76 & 2.16 \\
\hline$\%$ Foreign-born & 11.62 & 11.56 \\
\hline \% Refugees & 13.02 & 7.38 \\
\hline$\%$ Not proficient in English/French & 1.07 & 2.00 \\
\hline$\%$ Poor & 7.88 & 6.50 \\
\hline$\%$ Residents w/ college degree & 20.49 & 8.98 \\
\hline$\%$ Residents 65 years and older & 18.37 & 4.37 \\
\hline$\%$ Health-care workers & 13.40 & 3.13 \\
\hline$\%$ Agricultural workers & 3.90 & 3.09 \\
\hline Population density & 202.32 & 671.09 \\
\hline$N$ & \multicolumn{2}{|c|}{84} \\
\hline \multicolumn{3}{|l|}{ B. Characteristics of pseudo-CSDs } \\
\hline$\%$ Black & 0.78 & 1.31 \\
\hline$\%$ Foreign-born & 8.08 & 8.10 \\
\hline$\%$ Refugees & 7.92 & 9.75 \\
\hline$\%$ Not proficient in English/French & 0.45 & 0.97 \\
\hline$\%$ Poor & 5.07 & 2.82 \\
\hline$\%$ Residents w/ college degree & 16.65 & 8.83 \\
\hline$\%$ Residents 65 years and older & 19.97 & 6.71 \\
\hline$\%$ Health-care workers & 12.36 & 3.95 \\
\hline$\%$ Agricultural workers & 6.17 & 6.96 \\
\hline Population density & 239.46 & 532.26 \\
\hline$\% C S D s$ & \multirow{2}{*}{\multicolumn{2}{|c|}{$\begin{array}{c}78.5 \% \\
1202\end{array}$}} \\
\hline$N$ & & \\
\hline
\end{tabular}

Sources: University of Toronto's COVID-19 Canada Open Data Working Group Dashboard and tabular data from Statistics Canada estimated from 2016 Canadian Census

Sample: 84 health regions in Canada; 1240 CSDs/CDs. 
Table S3. Poisson Regression Models Predicting COVID-19 Infection and Mortality Rates (Actual Percentages)

\begin{tabular}{|c|c|c|c|c|c|c|}
\hline \multirow{2}{*}{$\begin{array}{l}\text { Characteristics of health regions/ } \\
\text { /Unstandardized covariates }\end{array}$} & \multicolumn{3}{|c|}{ Infection rates } & \multicolumn{3}{|c|}{ Mortality rates } \\
\hline & IRR & & $\beta / \mathrm{se}$ & IRR & & $\beta / \mathrm{se}$ \\
\hline \multicolumn{7}{|l|}{ A. Rates } \\
\hline$\%$ Black & 1.39 & $* * *$ & 136.97 & 1.54 & $* * *$ & 39.07 \\
\hline$\%$ Foreign-born & 0.95 & $* * *$ & -48.95 & 0.94 & $* * *$ & -11.01 \\
\hline$\%$ Refugees & 1.01 & $* * *$ & 10.53 & 1.02 & $* * *$ & 5.33 \\
\hline$\%$ Neither proficient in English/French & 1.16 & $* * *$ & 25.53 & 1.10 & $* *$ & 3.01 \\
\hline$\%$ Poor & 1.01 & $* * *$ & 11.42 & 1.03 & $* * *$ & 8.03 \\
\hline$\%$ Residents w/ a college degree & 0.99 & $* * *$ & -10.98 & 0.98 & $* * *$ & -6.17 \\
\hline$\%$ Residents 65 years and older & 1.02 & $* * *$ & 10.88 & 1.18 & $* * *$ & 17.61 \\
\hline$\%$ Health-care workers & 1.00 & & 0.70 & 0.93 & $* * *$ & -4.05 \\
\hline$\%$ Agricultural workers & 0.92 & $* * *$ & -22.39 & 0.81 & $* * *$ & -11.34 \\
\hline Lower density (land size) & 1.00 & $* * *$ & -17.21 & 1.00 & $* * *$ & -5.44 \\
\hline Intercept & 0.00 & $* * *$ & -119.81 & 0.00 & $* * *$ & -45.94 \\
\hline Pseudo- $R^{2}$ & & 0.60 & & & 0.73 & \\
\hline \multicolumn{7}{|l|}{ B. Counts } \\
\hline$\%$ Black & 1.49 & $* * *$ & 170.85 & 1.72 & $* * *$ & 49.04 \\
\hline$\%$ Foreign-born & 0.96 & $* * *$ & -42.50 & 0.93 & $* * *$ & -13.39 \\
\hline$\%$ Refugees & 1.02 & $* * *$ & 24.47 & 1.02 & $* * *$ & 7.11 \\
\hline$\%$ Neither proficient in English/French & 1.09 & $* * *$ & 15.44 & 1.20 & $* * *$ & 5.90 \\
\hline$\%$ Poor & 1.05 & $* * *$ & 61.87 & 1.08 & $* * *$ & 21.07 \\
\hline$\%$ Residents w/ a college degree & 1.02 & $* * *$ & 24.69 & 1.01 & $*$ & 1.97 \\
\hline$\%$ Residents 65 years and older & 1.02 & $* * *$ & 7.13 & 1.18 & $* * *$ & 17.02 \\
\hline$\%$ Health-care workers & 0.92 & $* * *$ & -26.39 & 0.85 & $* * *$ & -10.15 \\
\hline$\%$ Agricultural workers & 0.90 & $* * *$ & -30.51 & 0.79 & $* * *$ & -13.68 \\
\hline Lower density (land size) & 1.00 & $* * *$ & -12.06 & 1.00 & $* * *$ & -5.21 \\
\hline Intercept & 407.19 & $* * *$ & 106.40 & 4.61 & $* * *$ & 6.38 \\
\hline Pseudo- $R^{2}$ & & 0.72 & & & 0.83 & \\
\hline
\end{tabular}

Source: University of Toronto's COVID-19 Canada Open Data Working Group Dashboard and tabular data from Statistics Canada estimated from 2016 Canadian Census

Sample: 84 health regions in Canada

Notes: Offsets for population size. $* * * p<0.001 ; * * \mathrm{p}<0.01 ; * \mathrm{p}<0.05$ 
Table S4. Poisson Regression Models Predicting COVID-19 Infection and Mortality Rates (Standardized Covariates)

\begin{tabular}{|c|c|c|c|c|c|c|}
\hline \multirow{2}{*}{$\begin{array}{r}\text { Characteristics of health regions/ } \\
\text { /Standardized coefficients }\end{array}$} & \multicolumn{3}{|c|}{ Infection rates } & \multicolumn{3}{|c|}{ Mortality } \\
\hline & IRR & & $\beta / \mathrm{se}$ & IRR & & $\beta / \mathrm{se}$ \\
\hline \multicolumn{7}{|l|}{ A. April 15, 2020} \\
\hline$\%$ Black & 1.72 & $* * *$ & 72.22 & 2.02 & $* * *$ & 16.30 \\
\hline$\%$ Foreign-born & 0.59 & $* * *$ & -28.71 & 0.74 & ** & -2.76 \\
\hline \% Refugees & 1.08 & $* * *$ & 10.28 & 1.20 & $* * *$ & 5.08 \\
\hline$\%$ Neither proficient in English/French & 1.35 & $* * *$ & 17.66 & 1.23 & & 1.75 \\
\hline$\%$ Poor & 1.05 & $* * *$ & 6.35 & 1.20 & $* * *$ & 4.47 \\
\hline$\%$ Residents w/ college & 0.95 & $* * *$ & -5.38 & 0.76 & $* * *$ & -4.99 \\
\hline$\%$ Residents 65 years and older & 1.24 & $* * *$ & 16.41 & 2.48 & $* * *$ & 13.38 \\
\hline$\%$ Health-care workers & 0.98 & & -1.47 & 0.84 & & -1.92 \\
\hline$\%$ Agricultural workers & 0.71 & $* * *$ & -21.30 & 0.67 & $* * *$ & -4.66 \\
\hline Lower density (land size) & 0.92 & $* * *$ & -6.06 & 0.70 & $* *$ & -3.18 \\
\hline Intercept & 0.00 & $* * *$ & -719.51 & 0.00 & $* * *$ & -153.87 \\
\hline Pseudo- $R^{2}$ & & 0.60 & & & 0.50 & \\
\hline \multicolumn{7}{|l|}{ B. June 15,2020} \\
\hline$\%$ Black & 2.05 & $* * *$ & 155.98 & 2.75 & $* * *$ & 48.87 \\
\hline$\%$ Foreign-born & 0.52 & $* * *$ & -58.07 & 0.40 & $* * *$ & -17.79 \\
\hline$\%$ Refugees & 1.04 & $* * *$ & 8.51 & 1.07 & $* * *$ & 3.52 \\
\hline$\%$ Neither proficient in English/French & 1.53 & $* * *$ & 43.09 & 1.59 & $* * *$ & 8.70 \\
\hline$\%$ Poor & 1.01 & & 1.90 & 1.18 & $* * *$ & 7.51 \\
\hline$\%$ Residents w/ college degree & 0.90 & $* * *$ & -16.73 & 0.83 & $* * *$ & -7.87 \\
\hline$\%$ Residents 65 years and older & 1.21 & $* * *$ & 23.51 & 2.22 & $* * *$ & 22.33 \\
\hline$\%$ Health-care workers & 0.92 & $* * *$ & -8.92 & 0.81 & $* * *$ & -4.89 \\
\hline$\%$ Agricultural workers & 0.72 & $* * *$ & -32.18 & 0.41 & $* * *$ & -16.79 \\
\hline Lower density (land size) & 0.85 & $* * *$ & -17.62 & 0.70 & $* * *$ & -5.18 \\
\hline Intercept & 0.00 & $* * *$ & -995.45 & 0.00 & $* * *$ & -242.12 \\
\hline Pseudo- $R^{2}$ & \multirow{2}{*}{\multicolumn{3}{|c|}{0.63}} & \multirow{2}{*}{\multicolumn{3}{|c|}{0.74}} \\
\hline$N$ & & & & & & \\
\hline
\end{tabular}

Source: University of Toronto's COVID-19 Canada Open Data Working Group Dashboard and tabular data from Statistics Canada estimated from 2016 Canadian Census Sample: 84 health regions in Canada

Notes: Offsets for population size. ${ }^{* * *} \mathrm{p}<0.001 ; * * \mathrm{p}<0.01 ; * \mathrm{p}<0.05$ 
Table S5. Poisson Regression Models Predicting COVID-19 Infection and Mortality Rates (Standardized Covariates)

\begin{tabular}{lccc|ccc}
\hline Characteristics of health regions/ & \multicolumn{3}{c}{ Infection rates } & \multicolumn{3}{c}{ Mortality rates } \\
\cline { 2 - 7 } /Standardized coefficients & IRR & & $\beta /$ se & IRR & \multicolumn{3}{c}{$\beta /$ se } \\
\hline A. Main text & & & & & & \\
\% Black & 2.04 & $* * *$ & 138.16 & 2.56 & $* * *$ & 39.08 \\
\% Foreign-born & 0.53 & $* * *$ & -50.26 & 0.51 & $* * *$ & -11.03 \\
\% Refugees & 1.05 & $* * *$ & 9.40 & 1.13 & $* * *$ & 5.31 \\
\% Neither proficient in English/French & 1.35 & $* * *$ & 26.70 & 1.22 & $* *$ & 3.03 \\
\% Poor & 1.07 & $* * *$ & 11.45 & 1.23 & $* * *$ & 8.02 \\
\% Residents w/ college & 0.93 & $* * *$ & -10.96 & 0.84 & $* * *$ & -6.17 \\
\% Residents 65 years and older & 1.09 & $* * *$ & 10 & 2.08 & $* * *$ & 17.58 \\
\% Health-care workers & 1.01 & & 0.99 & 0.81 & $* * *$ & -4.04 \\
\% Agricultural workers & 0.78 & $* * *$ & -22.48 & 0.51 & $* * *$ & -11.35 \\
Lower density (land size) & 0.85 & $* * *$ & -17.19 & 0.64 & $* * *$ & -5.42 \\
Intercept & 0 & $* * *$ & -940.41 & 0 & $* * *$ & -211.34 \\
Pseudo- $R^{2}$ & & 0.60 & & & 0.73 & \\
B. Ontario and Quebec & & & & & & \\
\% Black & & & & & \\
\% Foreign-born & 1.99 & $* * *$ & 67.14 & 2.39 & $* * *$ & 22.28 \\
\% Refugees & 0.52 & $* * *$ & -35.49 & 0.71 & $* * *$ & -4.61 \\
\% Neither proficient in English/French & 0.88 & $* * *$ & -19.58 & 0.97 & & -1.12 \\
\% Poor & 1.40 & $* * *$ & 16.5 & 0.90 & & -1.27 \\
\% Residents w/ college degree & 1.23 & $* * *$ & 27.2 & 1.12 & $* * *$ & 4.16 \\
\% Residents 65 years and older & 0.74 & $* * *$ & -29.32 & 0.88 & $* *$ & -3.42 \\
\% Health-care workers & 1.06 & $* * *$ & 4.18 & 1.59 & $* * *$ & 7.95 \\
\% Agricultural workers & 1.16 & $* * *$ & 9.48 & 1.33 & $* * *$ & 3.95 \\
Lower density (land size) & 0.69 & $* * *$ & -25.88 & 0.74 & $* * *$ & -5.61 \\
Intercept & 0.51 & $* * *$ & -28.98 & 0.40 & $* * *$ & -6.70 \\
Pseudo- $R^{2}$ & 0.00 & $* * *$ & -653.12 & 0.00 & $* * *$ & -174.38 \\
& & 0.67 & & & 0.71 & \\
\hline
\end{tabular}

Source: University of Toronto's COVID-19 Canada Open Data Working Group Dashboard and tabular data from Statistics Canada estimated from 2016 Canadian Census

Sample: 84 health regions in Panel A; 52 health regions in Ontario and Quebec in Panel B Notes: Offsets for population size. $* * * p<0.001 ; * * p<0.01 ;{ }^{*} \mathrm{p}<0.05$ 